\title{
Moyens de calculs opérationnels de la dispersion atmosphérique
}

\author{
A. DOURY \\ (Manuscrit recu le 7 septembre 1981) \\ RÉSUMÉ
}

Sur le plan des nécessités opérationnelles, notamment aux stades les plus importants de la conception des ouvrages et de la prévention des configurations exceptionnelles de fonctionnement, il n'est pas possible d'éviter d'avoir recours à des moyens d'évaluation numérique de la dispersion atmosphérique.

En s'appuyant sur les pratiques les plus récentes de l'Institut de protection et de sûreté nucléaire du CEA, on tente de montrer que la situation actuelle pour un grand nombre d'utilisateurs n'est pas aussi inconfortable qu'on pourrait le craindre.

Après une présentation rapide de quelques moyens opérationnels confirmés, et une indication de leurs limites d'utilisation, on propose quelques formules extrêmement simplifiées destinées à fournir rapidement, en l'absence de moyens informatiques et de données météorologiques précises, des ordres de grandeurs utiles soit pour la validation approximative rapide d'un modele, soit pour l'action en situation exceptionnelle.

\section{ABSTRACT}

At the operational level, especially at the most important stages of plant design and prevention of exceptional operating circumstances, the use of tools to assess atmospheric dispersion quantitatively i.e. models - cannot be avoided. On the basis of the latest practices developed at the CEA, it is demonstrated that the present situation is not so uncomfortable for a large number of users as it could be feared. Some confirmed operational methods are presented together with their limits, and a few simplified equations are given in order to quickly supply useful orders of magnitude either for a quick approximative validation of a model or for emergency action when computing means and precise meteorological data are lacking.

\section{INTRODUCTION}

L'abondance quelque peu demesurée des publications concernant la "modélisation", c'est-à-dire, plus exactement, les moyens de calcul de la dispersion atmosphérique ainsi que l'apparition quasi continue de "nouveaux modèles", justifient peut-être une tentative de clarification qui sera présentée ici à la lumière des pratiques actuelles de l'Institut de protection et de sûreté nucléaire du CEA dans sa mission essentielle d'"appui technique de la puissance publique". Dans cette présentation, il sera tenu compte, en outre, des aspects de disponibilité et de limite d'utilisation des outils en cause.

* Commissariat à l'énergie atomique, Institut de protection et de sûreté nucléaire, BP 6, 92260 Fontenay-aux-Roses. 


\section{A. DOURY}

Le problème central est d'évaluer quantitativement, dans le temps et dans l'espace, en fonction d'une source donnée, des concentrations de polluants minoritaires passifs à partir desquelles il est ensuite possible, compte tenu de tous les processus physiques ou physicochimiques secondaires intervenant, de procéder aux calculs complets qui permettent d'aboutir aux détriments finaux.

Notre expérience dans ce domaine remonte à la fin des années 1950 , où se situe, en octobre 1957 à Windscale en Grande-Bretagne, le premier et unique accident de l'âge nucléaire dont les conséquences sur l'environnement aient comporté une contamination radioactive significative de l'atmosphère justifiant réellement l'utilisation de moyens d'évaluation quantitative. Par la suite, les impératifs de la sécurité radiologique de l'espace aérien pendant les essais nucléaires du Sahara et du Pacifique au cours des années 1960 et 1970 ont été une occasion d'enrichissement de cette expérience, et plus particulièrement d'extension à des échelles de temps et d'espace tout à fait différentes.

Cette notion d'échelle est fondamentale. Elle conduit généralement à distinguer trois domaines dans lesquels les problèmes et les difficultés ne sont ni de même nature, ni de même importance. Ces trois domaines comportent deux extrêmes dont l'existence est intuitive, le champ proche dominé par les conditions initiales et le champ lointain ou grandes distances. Entre ces deux extrêmes, on est obligé de considérer au moins un troisième domaine ou domaine intermédiaire souvent appelé méso-scalaire par les météorologistes.

On sait, par ailleurs, que les processus de transferts de polluants au stade de constituants minoritaires de l'atmosphère sont principalement gouvernés, dans des conditions qui dépendent précisément du domaine d'échelle considéré, par la physicochimie au niveau de ces constituants et par la météorologie. On peut dire qu'aux courtes distances et aux faibles durées de transfert, la physicochimie au niveau du constituant joue le rôle essentiel tandis que la météorologie ne pose pas de problème insurmontable. Aux grandes distances, au contraire, c'est la météorologie synoptique et la trajectographie résultante qui dominent, mais toujours sans poser de problème particulièrement crucial [2]. C'est véritablement dans le domaine intermédiaire, entre quelques dizaines et quelques centaines de kilomètres, que les plus grandes difficultés et les problèmes encore non résolus semblent se présenter avec le plus d'acuité, notamment au niveau des trajectoires qui s'avèrent plus complexes, en grande partie à cause de la nature même des écoulements atmosphériques à cette échelle en présence du relief.

Les problèmes purement météorologiques étant considérés comme résolus par des moyens spécifiques en liaison étroite avec les spécialistes concernés, il est souvent suffisant, pour les applications pratiques, de traiter sur trajectoire supposée connue, les processus d'évolution physicochimique du poliuant, parmi lesquels le plus important est probablement le processus physique de diffusion turbulente, qui dépend aussi de quelques paramètres météorologiques.

Dans cette optique, le point de départ est le bilan local de matière polluante, ou équation différentielle classique de transport-diffusion, et la solution passe par une distribution spatiale gaussienne de chaque échelon d'émission quasi ponctuel et quasi instantané. Des variantes peuvent être introduites, notamment pour la dimension verticale où l'hypothèse gaussienne est sûrement la plus critiquable, mais une attention constante doit être apportée au rendement basé sur le rapport performancescomplications, compte tenu principalement de la disponibilité réelle des données d'entrée, notamment météorologiques, dont le nombre et la qualité augmentent rapidement avec la complication du "modèle".

C'est pour rendre service aux utilisateurs éventuellement démunis de ces données, et pour obtenir rapidement des ordres de grandeur de fourchettes de résultats qui sont plus stables que ne pourrait l'imaginer le profane, que quelques formules ultra simples, rationnellement déduites des modèles et largement confrontées avec l'expé- 
rience, sont indiquées à titre complémentaire. Ces formules qui ne dépendent plus que de la distance parcourue par les polluants ne s'appliquent naturellement qu'à des configurations de rejet et de transfert suffisamment simples.

\section{L'EQUATION DE TRANSPORT-DIFFUSION}

La plupart des "modèles" proposés partent du bilan local de matière polluante - ou équation différentielle de transport-diffusion (ou advection-diffusion) - dont la version la plus utilisée, limitée ou "fermée" au second ordre, est :

$$
\frac{\delta x}{\delta t}=T+D+S+P
$$

$T=-\vec{v} \cdot \vec{\nabla}_{\chi}$ terme TRANSPORT ou ADVECTION

$\left.\mathrm{D}=\vec{\nabla} \overrightarrow{(\mathrm{K}} \cdot \vec{\nabla}_{\chi}\right)$ terme DIFFUSION TURBULENTE

$\mathrm{S}=-\vec{\nabla} \cdot \Phi_{\mathrm{S}}$ terme SOURCES

$P=-\vec{\nabla} \cdot \Phi_{p}$ terme PUITS

$\chi=$ concentration volumique du polluant minoritaire

$\stackrel{\mathrm{t}}{\rightarrow}=$ temps

$\vec{V}_{\vec{V}}=$ vitesse moyenne de l'écoulement porteur

$\overrightarrow{\mathrm{K}}=$ tenseur de diffusivité turbulente

$\Phi_{\mathrm{S}}=$ flux de sources

$\Phi_{p}=$ flux de pertes ou de puits

$\vec{\nabla}=$ opérateur de différentiation spatiale.

Dans les termes "puits" et "sources" figurent tous les processus secondaires susceptibles d'intervenir, tels que lavages par les précipitations atmosphériques, dépôts secs ou précipités, remises en suspension, décroissances radioactives, évolutions physicochimiques, qui sont traités par des modèles spécifiques complémentaires.

\section{TRAITEMENT NUMERIQUE DIRECT DE L'EQUATION DE TRANSPORT-DIFFUSION}

Sauf pour quelques configurations très simples, il est généralement impossible d'établir des solutions analytiques exactes qui satisfassent l'équation 
de transport-diffusion, même limitée au second ordre. C'est pourquoi des méthodes numériques directes sont souvent proposées, où des différences ou éléments finis, obtenus par des techniques de "discrétisation", remplacent les termes différentiels de l'équation initiale.

De telles méthodes nécessitent naturellement des moyens de calculs non négligeables. Elles sont aussi assez exigeantes sur le plan des données d'entrée concernant le champ météorologique de mouvement du fluide porteur d'une part, et les champs physiques des composantes du tenseur de diffusivité turbulente d'autre part. Si ces exigences sont contournées par des simplifications abusives, on peut se retrouver dans une situation où le traitement numérique proprement dit perd tout intérêt devant des solutions analytiques plus faciles à manipuler et que, précisément, les simplifications permettent.

II faut, cependant, reconnaître que si les questions posées impliquent obligatoirement des configurations compliquées et de nombreux processus d'évolution physicochimique du polluant, le problème ne peut en toute rigueur être traité que numériquement ou réputé insoluble. Certains écoulements méso-scalaires sont des exemples typiques de telles configurations compliquées, en présence de relief ou en bordure de grandes étendues d'eau, par exemple.

\section{SOLUTIONS ANALYTIQUES DE L'EQUATION DE TRANSPORT-DIFFUSION}

La configuration la plus simple que l'on puisse rencontrer est celle de l'émission quasi ponctuelle et quasi instantanée, en atmosphère libre, dégagée du sol et des obstacles, en mouvement moyen non nécessairement permanent mais uniforme, avec des propriétés diffusives elles-mêmes non nécessairement permanentes mais uniformes.

Dans ces conditions, il existe sur la trajectoire supposée connue du centre des masses de matières émises, en l'absence de tous autres processus de "puits" ou "sources", une solution analytique trigaussienne, également la plus simple et physiquement la plus probable :

$\chi=\Delta Q(2 \pi)^{-3 / 2}\left(\sigma_{x} \sigma_{y} \sigma_{z}\right)^{-1} \exp \left[-\frac{1}{2}\left(\frac{\left[x-\bar{u}\left(t-t_{e}\right)\right]^{2}}{\sigma_{x}^{2}}+\frac{y^{2}}{\sigma_{y}^{2}}+\frac{z^{2}}{\sigma_{z}^{2}}\right)\right]$ où :

$\Delta \mathrm{Q}=$ quantité émise

$\sigma_{x} \sigma_{y} \sigma_{z}=$ écarts-types de distribution spatiale des matières émises

$\overline{\mathrm{u}}=$ vitesse moyenne du vent

$x y z=$ coordonnées spatiales classiques avec origine au point source

$t_{e}=$ instant d'émission 
à condition que les écarts-types de cette solution, qui est une expression de la concentration de polluant répartie dans l'espace et évoluant dans le temps, satisfassent à certaines relations avec les diffusivités de l'équation différentielle initiale :

$$
\begin{aligned}
& \mathrm{K}_{\mathrm{ij}}=0 \text { pour } \mathrm{i} \neq \mathrm{j} \\
& \mathrm{K}_{\mathrm{iit}}=\frac{1}{2} \cdot \frac{\mathrm{d}}{\mathrm{dt}}\left(\sigma_{\mathrm{it}}^{2}\right)
\end{aligned}
$$

où :

ij $=$ indices de direction spatiale

$\mathrm{K}_{\mathrm{ij}}=$ diffusivité, fonction des fluctuations de vitesse du vent et de l'échelle de temps correspondante, contenue dans (1).

Les écarts-types sont avantageusement exprimés en fonction de la durée de transfert. Ils dépendent, en outre, éventuellement de la vitesse du vent, et pour l'écart-type vertical, de la stabilité du fluide atmosphérique porteur. Des valeurs opérationnelles de ces écarts-types, de nature purement expérimentale (traceurs), sont fournies par la formule (5) et les tableaux I et II:

$$
\sigma_{i t}=\left[A_{i}\left(t-t_{e}\right)\right]^{k_{i}}
$$

où :

$A_{i}=$ pseudo-coefficient de diffusion, constant

$\mathrm{k}_{\mathrm{i}}=$ exposant sans dimension, constant.

\section{REMARQUE IMPORTANTE}

II s'agit de valeurs moyennes temporelles considérées sur la même durée que les autres paramètres, notamment le vent et la concentration recherchée elle-même. Cette durée est précisément la durée de transfert entre la source et l'objectif, avec toutefois une limite inférieure de l'ordre de $10 \mathrm{~min}$ (moyenne internationale concertée pour une observation météorologique) et une limite supérieure de l'ordre de $3 \mathrm{~h}$ (plus petit intervalle synoptique concerté entre deux observations météorologiques). Le non-respect d'une telle pratique risquerait d'entraîner des interférences fâcheuses entre les domaines moyen et turbulent des processus.

TABLEAU ।

Diffusion normale

\begin{tabular}{|c|c|c|c|c|}
\hline $\begin{array}{c}\text { Durée de transfert } \\
\qquad \text { (secondes) }\end{array}$ & $\begin{array}{c}A_{x}=A_{y} \\
\left(m^{1 / k} \cdot s^{-1}\right)\end{array}$ & $\begin{array}{c}A_{z} \\
\left(m^{1 / k} \cdot s^{-1}\right)\end{array}$ & $\begin{array}{c}k_{x}=k_{y} \\
\text { (sans dim.) }\end{array}$ & $\begin{array}{c}\mathrm{k}_{\mathrm{z}} \\
\text { (sans dim.) }\end{array}$ \\
\hline 0 à $2,40.10^{2}$ & $4,05 \cdot 10^{-1}$ & 0,42 & 0,859 & 0,814 \\
\hline $2,40 \cdot 10^{2}$ à $3,28 \cdot 10^{3}$ & $1,35.10^{-1}$ & 1,00 & 1,130 & 0,685 \\
\hline $3,28 \cdot 10^{3}$ à $9,70 \cdot 10^{4}$ & $1,35.10^{-1}$ & 20,00 & 1,130 & 0,500 \\
\hline $9,70 \cdot 10^{4}$ à $5,08 \cdot 10^{5}$ & $4,63 \cdot 10^{-1}$ & 20,00 & 1,000 & 0,500 \\
\hline $5,08 \cdot 10^{5}$ à $1,30 \cdot 10^{6}$ & 6,50 & 20,00 & 0,824 & 0,500 \\
\hline$>1,30.10^{6}$ & $2,00.10^{5}$ & 20,00 & 0,500 & 0,500 \\
\hline
\end{tabular}

(Gradient vertical de température $\leqslant-0,5 \circ \mathrm{C} / 100 \mathrm{~m}$ ) (Durée d'observation quasi instantanée) 
TABLEAU II

Diffusion faible

(Gradient vertical de température $>-0,5 \circ \mathrm{C} / 100 \mathrm{~m}$ )

(Durée d'observation quasi instantanée)

\begin{tabular}{|c|c|c|c|c|}
\hline $\begin{array}{c}\text { Durée de transfert } \\
\mathrm{t} \text { (secondes) }\end{array}$ & $\begin{array}{c}\mathrm{a}_{\mathrm{x}}=\mathrm{A}_{\mathrm{y}} \\
\left(\mathrm{m}^{1 / \mathrm{k}} \cdot \mathrm{s}^{-1}\right)\end{array}$ & $\begin{array}{c}\mathrm{A}_{z} \\
\left(\mathrm{~m}^{1 / k} \cdot \mathrm{s}^{-1} \text { ) }\right.\end{array}$ & $\begin{array}{c}\mathrm{k}_{\mathrm{x}}=\mathrm{k}_{\mathrm{y}} \\
\text { (sans dim.) }\end{array}$ & $\begin{array}{c}\mathrm{k}_{z} \\
\text { (sans dim.) }\end{array}$ \\
\hline $0 \quad$ à $2,40 \cdot 10^{2}$ & $4,05 \cdot 10^{-1}$ & 0,20 & 0,859 & 0,500 \\
$2,40.10^{2}$ à $9,70.10^{4}$ & $1,35 \cdot 10^{-1}$ & 0,20 & 1,130 & 0,500 \\
$9,70.10^{4}$ à $5,08.10^{5}$ & $4,63 \cdot 10^{-1}$ & 0,20 & 1,000 & 0,500 \\
$5,08.10^{5}$ à $1,30.10^{6}$ & 6,50 & 0,20 & 0,824 & 0,500 \\
$>1,30.10^{6}$ & $2,00 \cdot 10^{5}$ & 0,20 & 0,500 & 0,500 \\
\hline
\end{tabular}

Si la diffusion s'effectue dans une couche de mélange bien caractérisée et d'épaisseur $\mathrm{H}$ connue, l'écart-type vertical admet une valeur limite supérieure :

$$
\sigma_{z_{\lim }}=\frac{1}{\sqrt{2 \pi}} \mathrm{H}
$$

Ces valeurs ont fait l'objet de publications [3, 5] qui fournissent également une justification de la limitation des catégories de diffusion à une seule dans le plan horizontal et à deux dans la direction verticale, dans des conditions telles que les résultats fournis par la méthode ont une probabilité très voisine de l'unité d'encadrer les résultats observés.

Pour le domaine des courtes distances et des faibles durées de transfert, où les effets de rugosité au voisinage du sol provoquent une dispersion systématique des valeurs observées autour des valeurs indiquées par les tableaux, il est précisé que les valeurs de ces tableaux sont centrées sur le cas d'une rugosité moyenne d'un terrain dégagé et non construit en présence de vitesses du vent de l'ordre de 3 à $6 \mathrm{~m} \cdot \mathrm{s}^{-1}$.

II existe une autre configuration très simple et couramment rencontrée pour laquelle la concentration de polluant dans l'espace peut s'exprimer par une solution analytique directe de l'équation de transport-diffusion. Cette configuration correspond à la précédente avec, comme hypothèses supplémentaires, un débit permanent, et non plus instantané, de l'émission quasi ponctuelle, une vitesse du vent au moins de l'ordre de $2 \mathrm{~m} . \mathrm{s}^{-1}$ [6], et des conditions météorologiques permanentes au moins en moyenne.

Dans ces conditions, le défilement sur le point d'observation est assez rapide pour permettre à ce point l'affectation d'un jeu d'écarts-types constants et un repérage en distance sous le vent de la source par l'expression:

$$
\mathrm{x}=\overline{\mathrm{u}} \mathrm{t}
$$

et on peut écrire:

$$
x=\frac{d Q}{d t}\left(2 \pi \bar{u} \sigma_{y} \sigma_{z}\right)^{-1} \exp \left[-\frac{y^{2}}{2 \sigma_{y}^{2}}-\frac{z^{2}}{2 \sigma_{z}^{2}}\right]
$$


où :

$\frac{\mathrm{dQ}}{\mathrm{dt}}=$ débit constant de la source quasi ponctuelle.

L'expression (8) n'est autre que l'équation bien connue dite "panache" (The Gaussian Plume Model) de Pasquill-Gifford, où les écarts-types peuvent admettre les valeurs des tableaux I et II pourvu que la,durée d'observation du phénomène, qui est ici essentiellement permanent, ne dépasse pas quelques minutes [5].

\section{SOLUTION MIXTE ANALYTIQUE-NUMERIQUE DE L'EQUATION DE TRANSPORT-DIFFUSION}

Une configuration plus complexe, mais cependant très importante, notamment en cas de situation exceptionnelle de rejet non concerté, est celle qui, dans le cadre des hypothèses de la première des deux solutions précédentes, avec des conditions météorologiques non permanentes, comporte en outre une caractéristique également non permanente de la cinétique du rejet que l'on suppose quelconque.

Dans ces conditions, le cas général de l'évolution historique de la concentration, essentiellement non permanente, est traité dans un deuxième temps par une intégration convenable de la solution analytique (2) relative à une émission instantanée. Sauf exception, cette intégration est impossible analytiquement. Elle est, par conséquent, remplacée par des traitements numériques.

La concentration en un point donné, à un instant donné, est ainsi obtenue pratiquement par une superposition numérique des effets simultanés de séquences d'échelons quasi instantanés simulant les émissions réelles et dont chaque centre de masses évolue naturellement sur sa trajectoire propre, supposée connue et définie comme un lieu de probabilité maximale de pollution (fig. 1). Cette opération équivaut à une intégration sur la durée d'émission.

Cette méthode présente notamment sur les méthodes traditionnelles du type Pasquill l'avantage de rester valable aux échelles supérieures où règne la turbulence "basse fréquence" et où la durée de transfert joue seule, ainsi qu'en cas de vent très faible où l'on retrouve des conditions de longues durées.

Elle convient, en outre, tout particulièrement au traitement des problèmes nombreux de "puits" et de "sources" en tout genre (radioactivité, physicochimie) où interviennent des fonctions de l'âge du polluant, c'est-à-dire précisément de la durée de transfert.

Le code de calcul TALD de Le Grand et Desprès [1] est une variante de ce modèle pour l'évaluation de concentrations moyennes annuelles en fonctionnement normal sur des distances supérieures à $100 \mathrm{~km}$ où dans ces conditions, la séquence d'émissions quasi instantanées est représentée par une série discrète biquotidienne, et l'expression des résultats en concentrations intégrées sur la durée d'exposition, avec une influence peu significative des vents faibles sur les moyennes annuelles, permet de faire disparaître la diffusion longitudinale dans des intégrations analytiques où apparaît un terme en $1 / \bar{u}$. 


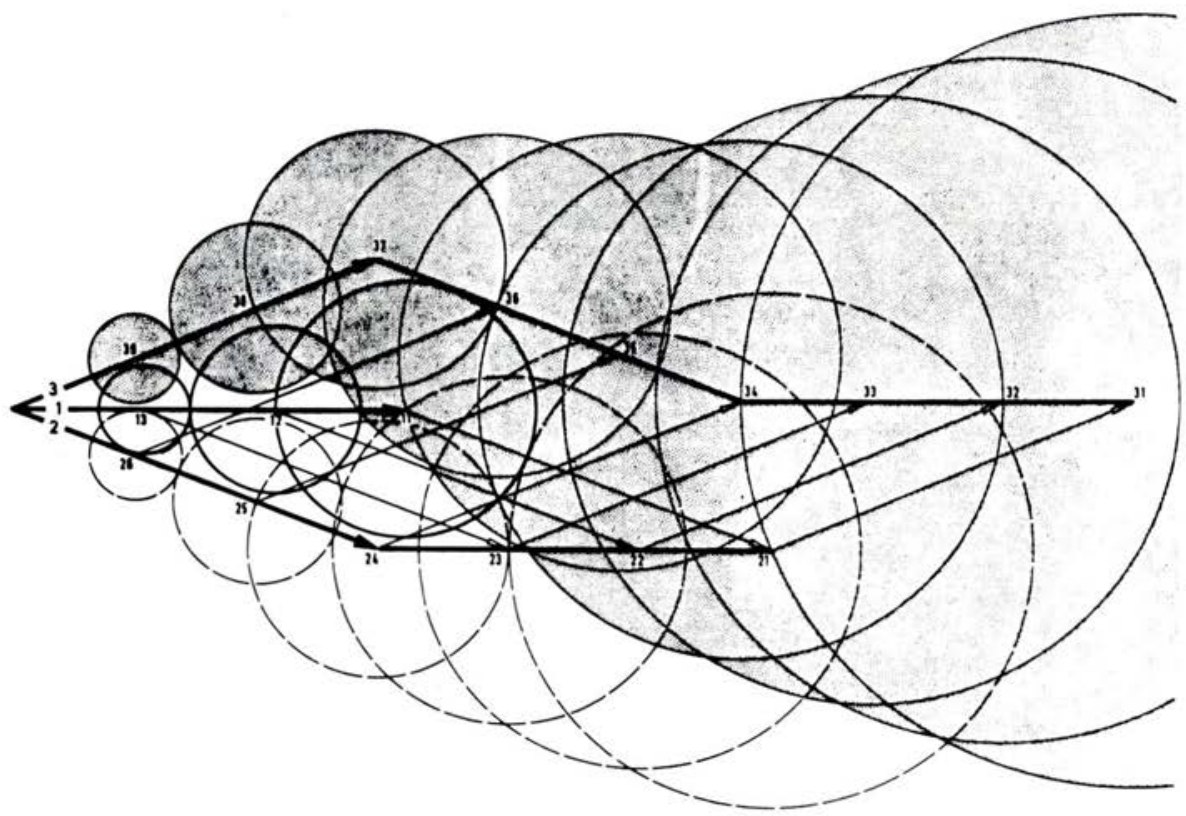

Fig. 1. - Principe de discrétisation d'une émission continue dans un fluide en mouvement moyen variable. Emission de 3, puis 6, puis 9 bulles. Trois configurations successives du panache résultant.

Les trajectoires des différents échelons sont calculées sur grille par un programme particulier qui prend en compte:

- le vent mesuré au point de rejet ;

- le vent moyen à $00 \mathrm{~h} 00$ et 12 h 00 T.U. en chaque point de la grille entre les surfaces $850 \mathrm{mb}$ et $1000 \mathrm{mb}$, fourni par les services météorologiques ;

- le vent moyen à $06 \mathrm{~h} 00$ et 18 h 00 T.U. aux mêmes points entre les mêmes surfaces, interpolé entre les situations à 00 h 00 et 12 h 00 T.U.

\section{LE COEFFICIENT DE TRANSFERT ATMOSPHERIQUE OU CTA}

Les équations appliquées au calcul de la concentration historique, en un point de l'espace, d'un polluant émis par une source quelconque, sont en fait des produits de convolution classiques entre une fonction "source" de l'instant d'émission, et une fonction "transfert" de la durée de transfert. Cette fonction "transfert" dépend des conditions dans le fluide vecteur (conditions météorologiques) qui se comporte comme un filtre "retard" et "passe-bas".

Si, d'autre part, et comme c'est fréquemment le cas dans la pratique, notamment en vue des calculs de doses d'exposition, on ne s'intéresse 
qu'à l'intégrale des conséquences sur la durée de l'exposition, il peut être suffisant, pour caractériser les conséquences, de connaître l'intégrale temporelle de la réponse historique en concentrations sur la totalité de la durée de passage sur l'objectif. II peut d'ailleurs arriver, notamment lorsque les cinétiques d'émission sont trop compliquées, que cette intégrale temporelle soit seule accessible.

Or, il se trouve $[4,5]$ que les fonctions "source", "transfert" et "concentration" sur la durée totale d'exposition sont reliées simplement par:

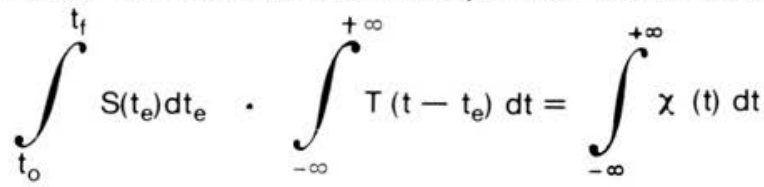

où :

$\mathrm{S}=$ fonction "source"

$T=$ réponse impulsionnelle de l'atmosphère (identiquement nulle pour $t-t_{e}<0$ ) dont la transformée de Laplace est la fonction de transfert classique

$\mathrm{t}_{\mathrm{e}}=$ instant d'émission à la source

$t_{\mathrm{o}}=$ instant de début d'émission

$t_{f}=$ instant de fin d'émission

$\mathrm{t}=$ instant d'observation des phénomènes

$x=$ concentration locale instantanée.

II en résulte que si l'on désigne par coefficient de transfert atmosphérique, ou CTA, l'intégrale, sur la durée de passage sur l'objectif, de la réponse impulsionnelle de l'atmosphère, on dispose alors d'une sorte d'invariant moyen, caractéristique des conditions moyennes de transfert et indépendant des "sources" et des "réponses", qui permet de passer automatiquement d'une quantité totale de polluant libéré à une concentration intégrée sur la durée totale d'exposition, soit :

$$
\text { C T A }=\frac{\int_{-\infty}^{+\infty} x(t) d t}{\int_{-\infty}^{+\infty} s\left(t_{e}\right) d t_{e}}
$$

En cas de régime permanent, le CTA prend la forme indéterminée $\frac{\infty}{\propto}$ qui tend vers une limite finie égale au quotient de la concentration constante par le débit constant.

Dans le cas des conditions de validité de l'équation (8), qui comprennent notamment une vitesse du vent au moins égale à $2 \mathrm{~m} \cdot \mathrm{s}^{-1}$, cette équation peut être utilisée pour une émission limitée, mais en ne fournissant plus qu'une valeur de CTA qui est alors équivalente au rapport : $x / \frac{\mathrm{dQ}}{\mathrm{dt}}$ 


\section{A. DOURY}

Des cahiers d’abaques précalculés sur ordinateur par la méthode des séquences d'émissions ponctuelles instantanées, ou méthode des "bouffées successives", ont été établis pour aider à la résolution rapide de problèmes courants [7]. Ces abaques fournissent systématiquement des CTA, ou concentrations intégrées pour des émissions unitaires (fig. 2). Des cartes de courbes d'iso-valeurs préparées en vue de situations exceptionnelles peuvent en être facilement déduites (fig. 3).

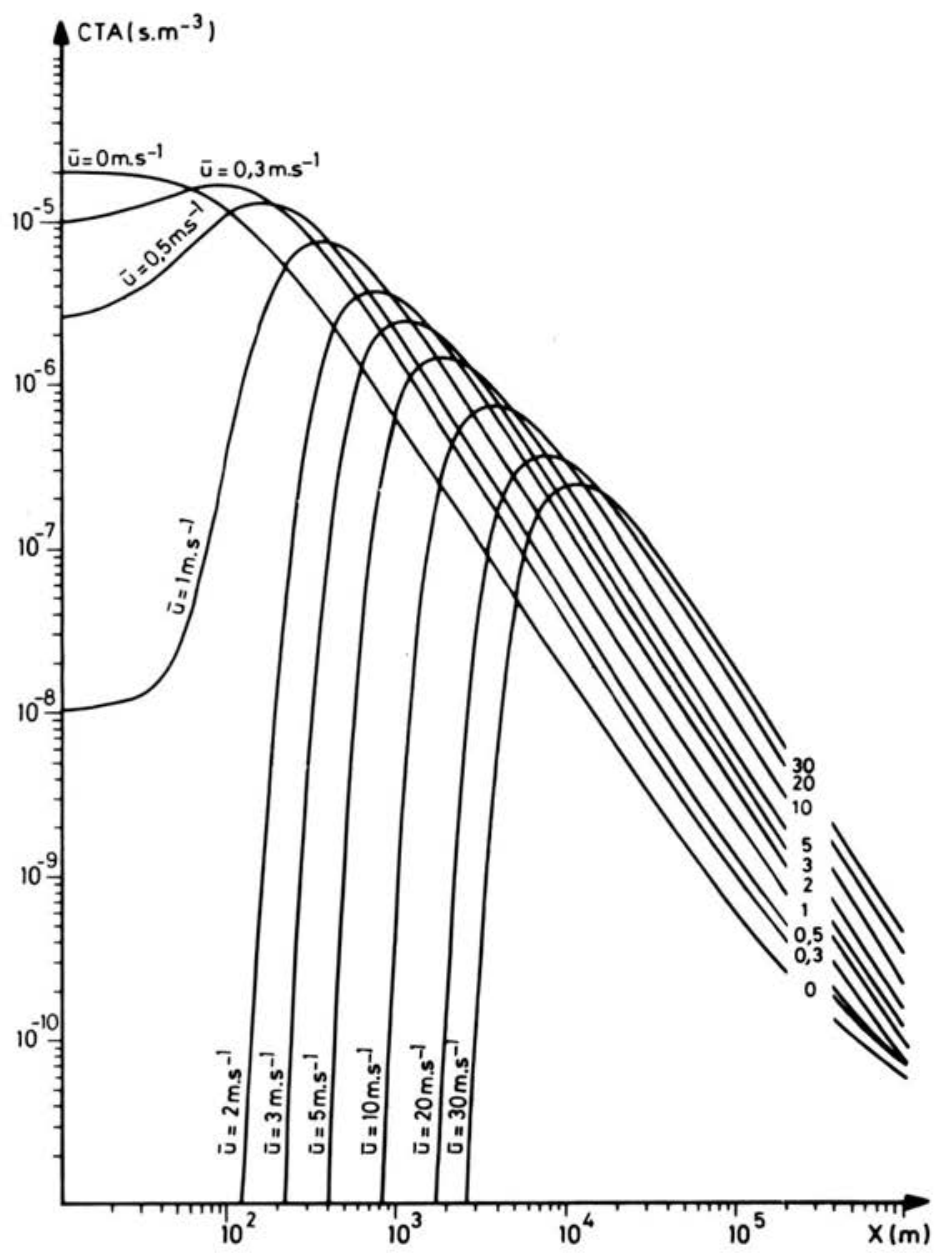

Fig. 2. - Coefficient de transfert atmosphérique maximal au sol en fonction de la distance sous le vent d'une émission ponctuelle de hauteur effective $100 \mathrm{~m}$. (Conditions atmosphériques : diffusion normale - vents de 0 à $30 \mathrm{~m}$. $\mathbf{s}^{-1}$ ). 


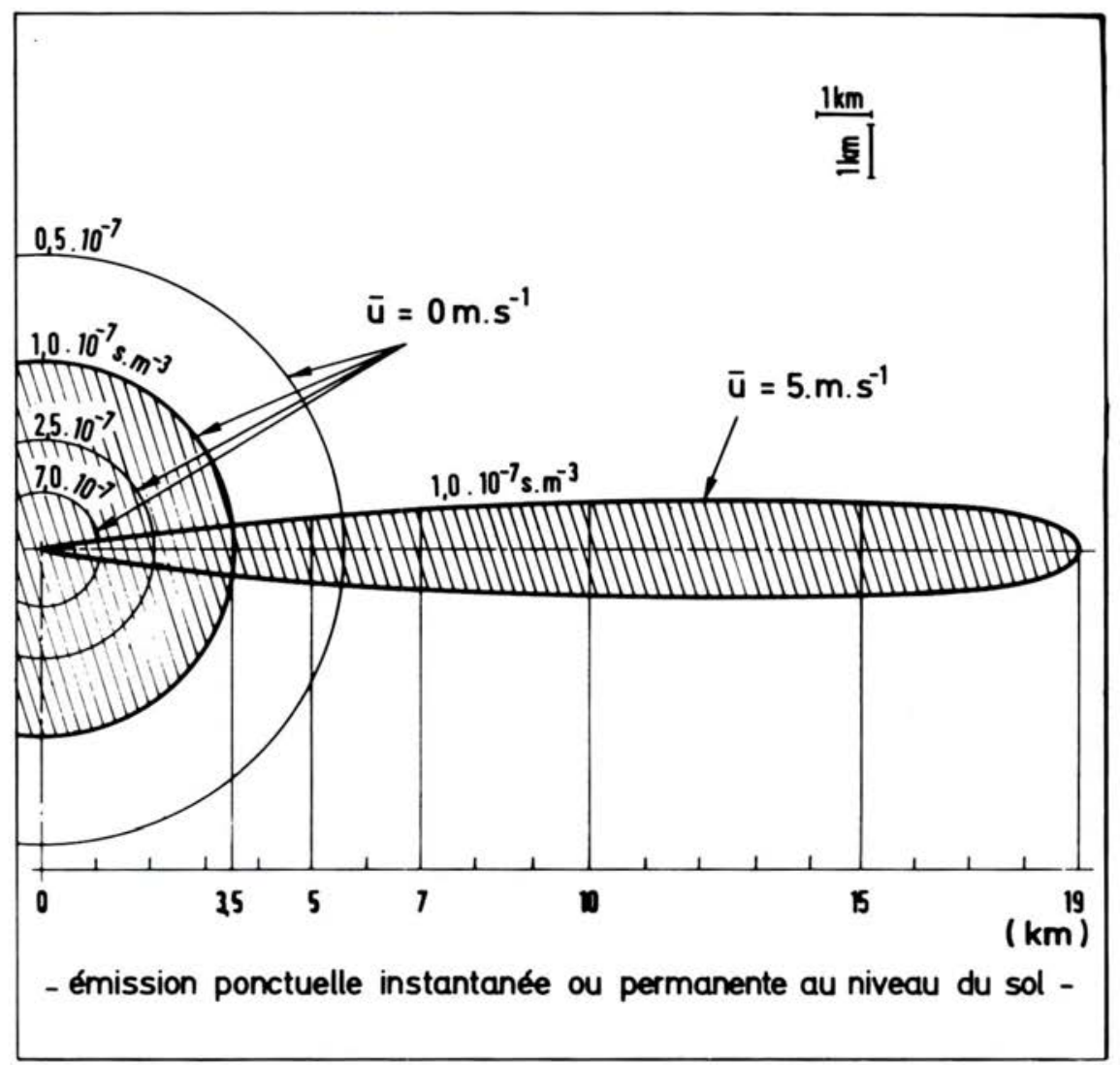

Fig. 3. - Exemple de schémas de principe de pollution atmosphérique, par vent nul ou établi dans des conditions simples.

\section{QUELQUES FORMULES ULTRA SIMPLES}

Après avoir constaté sur de nombreux exemples ou cas réels suffisamment simples et caractéristiques que les données d'observation s'accordaient généralement avec les résultats de calculs fournis par différents modèles, autour de valeurs présentant une dispersion plus limitée que celle à laquelle on était en droit de s'attendre, et que certains paramètres, notamment météorologiques, ne semblaient pas peser sur ces valeurs avec toute l'importance prévue, il nous a paru utile de faire connaître un certain nombre de relations ultra simplifiées, choisies parmi celles qui nous servent notamment pour pratiquer des vérifications rapides dans des mémoires de calcul, et dont la formulation même suggère les intervalles de confiance.

Il est important de signaler que ces formules ne proviennent pas seulement d'ajustements plus ou moins artificiels des résultats expérimentaux qui ont 
servi à les tester. Elles sont également déduites de modèles sérieux appliqués aux configurations les plus simples avec un certain nombre d'hypothèses simplificatrices supplémentaires. Ces hypothèses supplémentaires sont de nature à mettre l'utilisateur en garde contre des applications abusives.

Ces formules sont les suivantes:

\section{Dimension horizontale d'un "nuage" de polluant}

Exprimée sous forme d'écart-type de distribution, la dimension horizontale d'un nuage de polluant minoritaire passif provenant d'une émission ponctuelle en atmosphère libre est assez bien approchée jusqu'à 5 à 6 jours de durée de transfert par la formule suivante due à Heffter [9] :

où :

$$
\sigma_{\mathrm{h}}=0,5 \mathrm{t}
$$

$\sigma_{\mathrm{h}}=$ écart-type de distribution horizontale du polluant en mètres

$\mathrm{t}=$ durée du transfert en secondes.

Au-delà de dix jours, il faut passer à une loi en $\mathrm{t}^{0,5}$.

\section{Dimension verticale d'un "nuage" de polluant}

En l'absence de toute autre information, une approximation de dimension verticale permettant de poursuivre des calculs en cas de mélange vertical quasi homogène sur des distances comprises entre $100 \mathrm{~m}$ et $100 \mathrm{~km}$ sous le vent de la source, peut être déduite de:

$$
\begin{array}{ll}
H=\frac{x}{10} & \text { (diffusion normale) } \\
H=\frac{x}{100} & \text { (diffusion faible) }
\end{array}
$$

où :

$\mathrm{H}=$ épaisseur de la couche homogène équivalente

$x=$ distance sous le vent de la source entre $100 \mathrm{~m}$ et $100 \mathrm{~km}$.

\section{Coefficient de transfert atmosphérique}

Le CTA peut être approché sur le lieu du centre des masses de polluant, entre $100 \mathrm{~m}$ et $100 \mathrm{~km}$ sous le vent de la source, par:

$$
\begin{array}{lll}
\text { CTA }=10 x^{-2} & \left(\text { s. } m^{-3}\right) & \text { (diffusion normale) } \\
\text { CTA }=100 x^{-2} & \left(\text { s. } m^{-3}\right) & \text { (diffusion faible) }
\end{array}
$$

où $x$ est exprimé en mètres.

La simplicité de ces formules qui ne comportent que le paramètre "distance" provient des deux circonstances suivantes introduites dans les hypothèses supplémentaires:

a) Deux effets contradictoires de la vitesse du vent, sur les dimensions horizontales du nuage et sur la vitesse de défilement, sont exactement compensés ;

b) Les dimensions horizontale et verticale sont exprimées par les relations (11) et (12), qui correspondent à des conditions météorolologiques moyennes neutres ou stables et ne prétendent pas rendre compte étroitement de toute situation réelle. 


\section{Dépôt sec}

A partir du CTA donné par la formule (13) associée à une valeur, très courante, de $0,01 \mathrm{~m} \cdot \mathrm{s}^{-1}$ pour la vitesse apparente de dépôt sec turbulent, ou par la formule (13') et une "vitesse" de dépôt sec dix fois plus faible [4], on obtient pour le dépôt sec maximal à la verticale du lieu du centre des masses de polluant entre $100 \mathrm{~m}$ et $100 \mathrm{~km}$ sous le vent de la source, en négligeant l'appauvrissement du nuage :

$$
\frac{\Omega \mathrm{s}}{\mathrm{Q}}=10^{-1} \mathrm{x}^{-2} \quad\left(\mathrm{~m}^{-2}\right)
$$

où $Q$ est la quantité totale du polluant libéré, et $\Omega_{\mathrm{s}}$ le dépôt sec total par unité de surface.

Les données d'observations sont plus dispersées autour des résultats fournis par cette formule que les données correspondantes autour de ceux de la formule précédente pour les CTA. Cette circonstance est liée principalement à la valeur de la vitesse de dépôt souvent mal connue et ellemême assez dispersée sous l'influence de nombreux paramètres physicochimiques.

\section{Dépôt précipité}

Toujours dans les mêmes conditions et sans appauvrissement préalable du nuage, c'est-à-dire pour le cas, qui est le plus sévère, d'une précipitation n'intervenant qu'à la verticale du point d'observation, on obtient pour le dépôt précipité maximal à la verticale du lieu du centre des masses de polluant, entre $100 \mathrm{~m}$ et $100 \mathrm{~km}$ sous le vent de la source:

$$
\frac{\Omega p}{Q}=10^{-4} R x^{-1} \quad\left(m^{-2}\right)
$$

où $R$ est l'intensité de la précipitation en $\mathrm{mm}^{\mathrm{h}} \mathrm{h}^{-1}$ et $\Omega_{\mathrm{p}}$ le dépôt précipité total par unité de surface.

Autour des résultats fournis par cette formule, les données d'observations sont mieux groupées que dans le cas du dépôt sec de la formule (14). Cette circonstance provient d'une meilleure connaissance et d'une plus faible dispersion des valeurs de constantes de lavage [9] que des valeurs de vitesses apparentes de dépôt sec, ainsi que de la disparition, au cours de l'intégration sur la verticale, de l'influence des conditions de stabilité verticale de l'atmosphère.

\section{CONCLUSION}

L'entreprise tentée ici est périlleuse dans la mesure où elle est susceptible de susciter de nombreuses critiques notamment pour ce qui concerne un certain excès de simplification et de schématisation.

Nous persistons, toutefois, à penser que les simplifications introduites sont non seulement souvent utiles mais aussi quelquefois nécessaires. II est, en effet, évident qu'en allant du plus simple au plus compliqué, les différents modèles ou méthodes de calculs sont de plus en plus exigeants 
pour ce qui concerne les données d'entrée, et que dans le domaine des calculs de dispersion atmosphérique, cette circonstance s'applique plus particulièrement aux données météorologiques dont les techniques et les coûts d'acquisition posent de nombreux problèmes.

$\mathrm{Si}$, dans cette optique et avec le souci de la performance dans l'économie et l'efficacité, on considère le cas, pas tout à fait improbable, où l'on ne dispose d'aucune information météorologique sérieuse, il peut arriver que les formules ultra simples proposées présentent un certain intérêt puisqu'elles ne comportent apparemment que des exigences de distances à l'exclusion de toute information de nature météorologique.

En y regardant de plus près, on constate, cependant, qu'il subsiste toujours une information météorologique strictement indispensable - celle du vent au niveau d'évolution des effluents rejetés - qui conditionne le seul paramètre des formules les plus simples possibles à savoir la distance, c'est-à-dire le positionnement dans l'espace ainsi que les délais de transfert. C'est pourquoi il nous semble que les efforts devraient être maintenant portés sur le problème de l'acquisition du vent au niveau d'évolution des produits rejetés, que ce soit par une instrumentation spécifique sur site ou par des modèles d'écoulement méso-scalaires.

De telles formules réduites à leur plus grande simplicité, certes incapables de traiter tous les problèmes mais plus facilement compréhensibles et discutables par le profane, peuvent présenter un autre intérêt en constituant un moyen de persuasion de certaines personnes qui continueraient à penser que, compte tenu notamment de la diversité des modèles proposés, l'évaluation numérique de la dispersion atmosphérique resterait, quelles que soient les configurations, une affaire hautement hasardeuse et non résolue.

\section{RÉFÉRENCES}

[1] DESPRES A., DOURY A., LE GRAND J. Le programme de calcul TALD pour l'évaluation des transferts à longue distance dans les basses couches de l'atmosphère. In : Les rejets radioactifs et leur dispersion dans l'atmosphère à la suite d'un accident hypothétique de réacteur, Ris $\varnothing, 22-25$ avril 1980. Luxembourg : Commission des communautés européennes, 1980, II, 743-775.

[2] DOURY A. Le problème des trajectoires atmosphériques à grande échelle traité par les équations d'énergie. La Météorologie, 1968, 11, 472-491.

[3] DOURY A. Une méthode de calcul pratique et générale pour la prévision numérique des pollutions véhiculées par l'atmosphère. Rapport CEA-R-4280, Rév. 1, 1976.

[4] DOURY A. Pratiques françaises en matière d'évaluation quantitative de la pollution

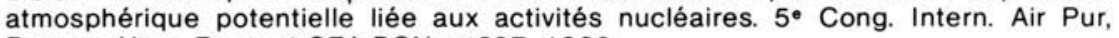
Buenos Aires, Rapport CEA-DSN n³97, 1980.

[5] DOURY A. Le Vadecum des transferts atmosphériques. Rapport CEA-DSN n 440 , 1981.

[6] DOURY A. Les limites d'utilisation des modèles "Panache" aux courtes distances et par vent faible. In : Les rejets radioactifs et leur dispersion dans l'atmosphère à la suite d'un accident hypothétique de réacteur, Ris $\phi, 22$ - 25 avril 1980. Luxembourg: Commission des communautés européennes, 1980, II, 615-648. 
MOYENS DE CALCULS OPÉRATIONNELS DE LA DISPERSION ATMOSPHÉRIQUE

[7] DOURY A., GERARD R., PICOL M. Abaques d'évaluation directe des transferts atmosphériques d'effluents gazeux. Rapport CEA-DSN n 84, Rév. 2, 1980.

[8] DOURY A. Prévision numérique des lavages et retombées dus aux précipitations atmosphériques. Rapport CEA-DSN n²92, 1979.

[9] HEFFTER J.L., TAYLOR A.D., FERBER G.J. A regional-continental scale transport, diffusion, and deposition model. NOAA-ERL-ARL 50, 1975. 